Sustinere

Journal of Environment and Sustainability

Volume 4 Issue 1 (2020) 16-23

Print ISSN: 2549-1245 Online ISSN: 2549-1253

Website: https://sustinerejes.com E-mail: sustinere.jes@iain-surakarta.ac.id

\title{
SHORT COMMUNICATION \\ Isolation and Characterization of Streptomycetes with Potential to Decompose Organic Compounds During Bioremediation of Arable Soil
}

\author{
Ekenwosu Joseph Ugochuwu*, Peter Ugochukwu Okorie \\ Department of Animal and Environmental Biology, Faculty of Science, Imo State University Owerri, \\ Nigeria \\ Article history: \\ Received 13 February 2020 | Accepted 24 April 2020 | Available online 30 April 2020
}

\begin{abstract}
The study has an objective of isolating and characterizing suspected Streptomycetes with the potential to decompose organic compounds in arable soil. The isolates were grown on a culture media and a total of 61 slopes were inoculated and labeled using the following characterization tests: catalase test, gram staining, oxidase test, motility test, and oxidativefermentative test. After characterization tests, data generated in the laboratory were analyzed and the study showed that isolates $A_{2}(c, e), B_{2}(c), C_{1}(b), C_{2}(a), D_{1}(e)$, and $D_{2}(d)$ were suspected to be Streptomycetes species. Isolates $\mathrm{D}_{1}(\mathrm{~d})$ and $\mathrm{D}_{2}(\mathrm{~b})$ were suspected to be Escherichia coli while isolates $\mathrm{A}_{1}(\mathrm{a}), \mathrm{B}_{2}(\mathrm{a}, \mathrm{b})$, and $\mathrm{D}_{1}(\mathrm{a})$ were suspected to be Bacillus species. Isolates $\mathrm{A}_{1}(\mathrm{~b}), \mathrm{C}_{1}(\mathrm{a}), \mathrm{D}_{1}(\mathrm{c})$, and $\mathrm{D}_{2}$ (e) were suspected to be Pseudomonas species. Further identification showed that isolates $\mathrm{A}_{2}$ (d) and $\mathrm{B}_{1}$ (b) could be Enterobacter species while isolates $\mathrm{A}_{2}(\mathrm{a}, \mathrm{b})$ were suspected to be Klebsiella species. The study tentatively identified Streptomycetes species; Escherichia coli; Bacillus species; Enterobacter species; Pseudomonas species and Klebsiella species. The suspected Streptomycetes identified were considered as potential organic matter decomposers in arable soil.
\end{abstract}

Keywords: Streptomycetes, Escherichia coli, Bacillus, Pseudomonas, Enterobacter, Klebsiella.

\section{Introduction}

During bioremediation, microorganisms degrade contaminants or organic substances to nontoxic products. The degradation of organic substances by soil microorganisms normally results in the production of nutrients such as nitrogen, potassium, and phosphorus (NPK) which in turn adds to soil fertility. However, the goal of bioremediation of arable soil is the stimulation of microorganisms to restore the soil's fertility. This technique relies on the activities of indigenous microorganisms by supplying them with nutrients needed for their metabolism. A wide range of microorganisms takes part in converting organic compounds into nutrients and

${ }^{*}$ Corresponding author. E-mail: josephekenwosu@gmail.com https://doi.org/10.22515/sustinere.jes.v4i1.97 
stable non-toxic products. In general, the metabolic activities of soil microorganisms make them an important component in the bioremediation process.

Streptomycetes universally occur in soil and water. Many species of these bacteria are important microorganisms involved in the degradation of organic matter in the soil thereby contributing to improving soil fertility by bioremediation. They are microbiologically characterized as gram-positive $(+)$ aerobic bacteria of complex form with a similar threadlike net like the fungi and bearing spores at maturity. This microorganism contributes to the earthy odour of soil and decaying leaves. Huddleston et al. (1997) reported that Streptomycetes could not be found in all soil like wet and cold soil but are abundant in dry and warm soil. The activities of Streptomycetes in the soil can be affected by pollutants and this has a multiplier effect on plant growth within the area. Alvarez et al. (1996) reported that out of four commercial composts added to tomato plants, three significantly improved plant growth, and one depressed it. They found out that the compost additions caused variations in the number of Streptomycetes, bacteria, and fungi in the rhizosphere. This can be attributed to the fact that soil samples from the site that inhibited plant growth had a high level of contaminants which significantly reduced the microbial population to less than $10^{3} \mathrm{CFU}$ per gram of soil. On the other hand, soil samples from the site that promoted plant growth had a microbial population that was not stressed. Anderson and Macfadyen (1975) also demonstrated that the soil microbial population is affected by the level of contaminants present in the soil.

\section{Literature review}

Several studies have shown that microorganisms play a crucial role in soil bioremediation process and subsequently improve plant growth and yield. In an investigation to screen off freeliving rhizospheric bacteria for their multiple plant growth-promoting activities. Ahmad et al. (2008) reported that rhizospheric bacteria showed great capacity to improve soil conditions thereby enhancing plant growth directly or indirectly. However, they must be in the right number to effectively degrade organic contaminants to non-toxic substances. Kumar et al. (2017) reported that microbes interact by physically or structurally altering the chemical composition of contaminants by enzymatic activity thereby reducing their toxicity. An experiment was conducted by Ekenwosu (2019) to study the post bioremediation effect of soil fertility using substrate from livestock byproduct. The study established that soil benefitted from livestock byproduct treatment enhanced soil microbial activities thereby significantly improving soil fertility. Huddleston et al., (1997) conducted an investigation to study the molecular detection of streptomycin producing streptomycetes in Brazilian soil. They established that actinomycetes isolated from soybean rhizosphere soil collected at two field sites were identified as Streptomycetes species. The research concluded that Streptomycetes present in soil rhizosphere were able to biodegrade organic compounds and also able to produce streptomycin. Similarly, in a paper titled "The role of terrestrial and aquatic organisms in decomposition processes", presented by Anderson and Macfadyen (1975) at the 17th symposium of the British Ecological Society, reported that soil microbial population is affected by the level of contaminants present in the soil. They reported that the population of Streptomycetes isolated from soil rhizosphere were more in number than those beneath the soil. They concluded that more Stretomycetes population density can be found at the top of the soil profile but as you go deep into the soil profile, their numbers are reduced. In another study, Osama et al. (2018) reported that the percentage of total microbial count decreased significantly because of the 
presence of Cadmium (a heavy metal) in the soil. They also reported that the decrease in soil microbial population negatively affected plant growth and essential minerals such as N, P, and K. Studies have shown that microbial degradation of organic contaminants has recorded huge success in the recovery of contaminated soil. In an investigation carried out by Das and Adholeya (2012) on the role of microorganisms in the remediation of contaminated soil, they reported that microorganisms help in the biodegradation of organic contaminants. Similarly, Srivastava et al. (2014) conducted an investigation to study advances in microbial bioremediation and factor influencing the process. They reported that soil microorganism is a factor to be considered in remediating contaminated soil. In their review, Ite and Ibok (2019) provided a clear insight into the role of microbes in microbial degradation of contaminated soil. They reiterated the role of plant-microbe interactions with respect to the biodegradation of contaminants in the soil. In an attempt to isolate and characterize filter paper degrading bacteria, Egwuatu and Appeh (2018) reported that bacteria cultures showed positive growth as the medium turned cloudy. They described Chryseobacterium luteola as having the highest degradation rate of $95 \%$ while Pseudomonas mendocina had a degradation rate of $90 \%$. Other microbes like Klebsiella terrigena, Burkholderia pseudomallei, and Klebsiella oxytoca all had a biodegradation rate of $75 \%$ each.

\section{Methods}

\subsection{Materials}

This study was carried out in the experimental farm, Imo State University Owerri located at latitude $5^{\circ} 30^{\prime} 13^{\prime \prime} \mathrm{N}$ and longitude $7^{\circ} 2^{\prime} 37^{\prime \prime} \mathrm{E}$ south-east Nigeria. Köppen's climate classification identified the study region as having the highest average temperature of $27.9^{\circ} \mathrm{C}$ in March while August remains the coldest month with an average temperature of $25.0^{\circ} \mathrm{C}$.

\subsection{Soil sample collection}

A combination of deep sampling, surface sampling, and composite sampling methods were adopted (Venosa et al., 1996). In this case, soil samples were collected at several points. These individual samples were joined to form a composite sample.

\subsection{Morphological characterization and isolation of organic compound decomposing microorganisms}

Bushnell Hass broth solution was prepared according to the manufacturer's instructions. Also, nutrient agar was prepared based on the instructions from the manufacturers to stimulate microbial growth. Stones, iron, dirt, etc were removed by handpicking from soil samples and then $200 \mathrm{~g}$ of the soil was placed in different conical flasks labeled A, B, C, and D. The culture was plated out on already prepared nutrient agar after 7 days and pure colonies obtained by subculturing. The morphological examination was conducted to ascertain the size, pigmentation, and shape of the colonies. Biochemical characteristics such as gram reaction, catalase test, oxidase test, and $\mathrm{O}-\mathrm{F}$ test were also carried out on isolates.

\subsubsection{Inoculation}

Using an aseptic technique, an inoculating loop was sterilized on the flame and then placed in conical flask A to collect a loop full of the solution which was then transferred to a portion of plate $A_{1}$ and $A_{2}$. While on the plates, streaks were made using standard streaking techniques such as the quadrant streak method to create a large surface area for microbial growth. The above 
procedure was carried out on other soil samples. After inoculation, plates were left in an incubator for suspected growth of microorganisms.

\subsubsection{Collection of isolates in pure culture (slopes)}

Each isolate was inoculated with a flame sterilized inoculating loop in already prepared agar medium (slopes). On the introduction, streaks were made on the slopes to give a large surface area using standard streaking techniques. Streaked slopes were stored in an incubator to observe microbial growth. A total of 61 slopes were inoculated and labeled.

\subsection{Smearing and gram staining of isolated colonies}

\subsubsection{Smearing}

A sterilized wire loop was used to get drops of distilled water and placed on a clean glass slide. The wire loop was sterilized a second time on the flame and then its edge used to collect a little portion of the isolate. This was disposed of on the glass slide by rubbing and then dried on the slide by passing it through the flame.

\subsubsection{Gram reactions}

Gram staining technique was used to determine the gram reactions. Already prepared smear was first of all treated with crystal violet which was subsequently washed off after 30 seconds. Smear was later treated with an iodine solution and then washed off with water after 30 seconds. Immediately ethyl alcohol was added on the smear, it was washed off and treated with safranin solution and allowed to act for 30 seconds before washing off. A drop of immersion oil was added on the slide and examined under the microscope.

\subsubsection{Catalase reaction}

Catalase test was used to demonstrate the presence of catalase, an enzyme found in microorganisms and capable of breaking down hydrogen peroxide into water and oxygen as seen in the equation:

$$
\mathrm{E}+2 \mathrm{H}_{2} \mathrm{O}_{2} \longrightarrow 2 \mathrm{H}_{2} \mathrm{O}+\mathrm{O}_{2}
$$

In the catalase reaction, $2 \mathrm{ml}$ of hydrogen peroxide solution was added into a test tube. Using aseptic technique, flame sterilized tweezers were used to take some pure cultures and placed on a dried glass slide. A drop of hydrogen peroxide was added on the glass slide. The appearance of gas bubbles indicates that the microorganism is catalase-positive $(+)$. On the other hand, the non-production of gas bubbles is an indication that the microorganism is catalase negative (-).

\subsubsection{Motility test}

Hanging drop method was used to determine the motility of cultured samples. This test was used in this study to differentiate microorganisms that are motile from those that are nonmotile. Two drops of liquid pure culture were placed on a clean cover slide. A cavity slide already greased with vaseline was turned over the cover slide. It was re-inverted and viewed under the microscope. 


\subsubsection{Oxidase test}

The oxidase test was used to identify microorganisms that produce the enzyme oxidase. In the study, a filter paper was carefully placed in a petri dish and some drops of tetramethyl-pphenylenediamine dihydrochloride solution were added to the filter paper containing smeared pure culture. A purple colour indicates a positive $(+)$ reaction i.e. presence of oxidase. However, the absence of purple colour indicates a negative $(-)$ reaction i.e. absence of oxidase.

\subsubsection{Oxidative-fermentative test ( $\mathrm{O}-\mathrm{F}$ test)}

This test was carried out to determine those microorganisms in the study area that are fermenters i.e. they can ferment glucose. The oxidative-fermentative medium was prepared in a beaker and upon the introduction of the indicator, the colour of the solution changed to red. The solution was poured into test tubes and carefully plugged with cotton wool and aluminium foil and then sterilized in an autoclave at $121{ }^{\circ} \mathrm{C}$ for 15 minutes. After 2 days, pure cultures were inoculated into the test tubes with the aid of a sterilized wire loop. Liquid paraffin was added to prevent the entrance of oxygen thereby providing an oxygen-free environment.

\section{Results}

The potential for Streptomycetes to contribute towards improving soil fertility by bioremediation is tremendous. This microorganism which contributes to the earthy odour of soil and decaying leaves is an important component of the soil ecosystem and they differ in biochemical activity and morphology. After culturing bacteria on nutrient agar, a result of the morphological examination was recorded as seen in Table 1. Biochemical characterization of pure isolates was carried out to tentatively identify the microorganisms therein as seen in Table 2. In this study, suspected Streptomycetes capable of decomposing organic compounds during bioremediation were tentatively identified. This is in agreement with earlier studies by Huddleston et al. (1997). They established that Streptomycetes present in the soil were able to biodegrade organic compounds. Other suspected microorganisms such as Escherichia coli, Bacillus species, Enterobacter species, Pseudomonas species, and Klebsiella species were also tentatively identified from the arable soil. The result from Table 2 revealed that isolates $\mathrm{A}_{2}$ (c and e), $B_{2}(c), C_{1}(b), C_{2}(a), D_{1}(e)$, and $D_{2}(d)$ could be Streptomycetes species because of the following reasons: they are gram-positive rods, they are aerobic, they are motile, they are catalase-positive, they are oxidase-positive, and they are fermenters.

Laboratory result from the study also revealed that isolates D1 (d) and D2 (b) may be Escherichia coli because they can ferment glucose, they are oxidase-negative, they are grampositive rods, they are catalase-positive, and they are motile. Further results revealed that isolates A1 (a), B2 (a, b), and D1 (a) could be some species from Bacillus group because they are catalase-positive, they are gram-positive rods, they are oxidase-positive, and they are fermenters.

In addition, the study also revealed that isolates A1 (b), C1 (a), D1 (c), and D2 (e), could be Pseudomonas species because they are catalase-positive, they are oxidase-positive, they are motile, they are gram-negative rods, and they are not fermenters. Enterobacter species were suspected from isolates A2 (d) and B1 (b) because they are gram-positive, they are motile, they are catalase-negative, they are fermenters, and they are oxidase-positive. Findings from the 
Table 1. Colonial morphology of bacteria on nutrient agar

\begin{tabular}{ccccccccc}
\hline Isolates & $\mathbf{A}_{\mathbf{1}}$ & $\mathbf{A}_{\mathbf{2}}$ & $\mathbf{B}_{\mathbf{1}}$ & $\mathbf{B}_{\mathbf{2}}$ & $\mathbf{C}_{\mathbf{1}}$ & $\mathbf{C}_{\mathbf{2}}$ & \multicolumn{1}{c}{$\mathbf{D}_{\mathbf{1}}$} & $\mathbf{D}_{\mathbf{2}}$ \\
\hline Shape & Circular & Circular & Rectangular & Circular & Circular & Circular & Rectangular & Circular \\
Size & Small & Medium & Large & Small & Small & Large & Medium & Small \\
Pigmentation & Cream & White & White & Cream & Cream & White & Cream & Cream \\
Elevation & Convex & Convex & Convex & Convex & Convex & Convex & Convex & Convex \\
Margin & $1 \mathrm{~mm}$ & $2 \mathrm{~mm}$ & $2.5 \mathrm{~mm}$ & $1.5 \mathrm{~mm}$ & $1 \mathrm{~mm}$ & $3 \mathrm{~mm}$ & $2.5 \mathrm{~mm}$ & $1 \mathrm{~mm}$ \\
\hline
\end{tabular}

Table 2. Biochemical characteristics of isolates

\begin{tabular}{|c|c|c|c|c|c|c|c|c|c|c|c|c|c|c|c|c|c|c|c|c|c|c|c|c|c|c|c|c|c|c|c|c|c|c|c|c|c|c|c|c|c|}
\hline \multirow{2}{*}{ Isolates } & \multicolumn{5}{|c|}{$\mathbf{A}_{1}$} & \multicolumn{5}{|c|}{$\mathbf{A}_{2}$} & \multicolumn{5}{|c|}{$\mathbf{B}_{1}$} & \multicolumn{5}{|c|}{$\mathbf{B}_{2}$} & \multicolumn{5}{|c|}{$\mathrm{C}_{1}$} & \multicolumn{5}{|c|}{$\mathbf{C}_{2}$} & \multicolumn{5}{|c|}{$\mathbf{D}_{1}$} & \multicolumn{6}{|c|}{$\mathbf{D}_{2}$} \\
\hline & $\mathbf{a}$ & $\mathbf{b}$ & c & d & $\mathbf{e}$ & $\mathbf{a}$ & b & c & d & $\mathbf{e}$ & $\mathbf{a}$ & b & $\mathbf{c}$ & d & e & $\mathbf{a}$ & b & c & d & $\mathbf{E}$ & $\mathbf{a}$ & $\mathbf{b}$ & c & d & $\mathbf{e}$ & $\mathbf{a}$ & B & $\mathbf{c}$ & d & $\mathbf{e}$ & $\mathbf{a}$ & $\mathbf{b}$ & c & d & & 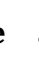 & a & $\mathbf{b}$ & c & d & e \\
\hline $\begin{array}{c}\text { Gram } \\
\text { reaction }\end{array}$ & + & - & - & - & + & - & - & + & - & + & - & - & - & + & - & + & + & + & - & - & - & + & - & - & - & + & - & - & - & - & + & - & - & - & & & - & - & - & + & - \\
\hline $\begin{array}{c}\text { Catalase } \\
\text { test }\end{array}$ & + & + & + & + & + & + & + & + & - & + & + & - & + & + & + & + & + & + & + & + & + & + & + & + & + & + & + & + & + & + & + & + & + & + & - & & + & + & + & + & + \\
\hline $\begin{array}{c}\text { Oxidase } \\
\text { test }\end{array}$ & + & + & + & + & - & + & + & + & + & + & + & + & + & + & + & + & + & + & + & + & + & + & + & + & + & + & + & + & + & + & + & + & + & - & - & + & + & - & + & + & + \\
\hline $\begin{array}{c}\text { Motility } \\
\text { test }\end{array}$ & + & + & + & - & - & - & - & + & + & + & + & + & - & - & + & - & + & + & + & - & + & + & + & + & + & + & + & + & + & + & - & + & + & + & - & + & + & + & - & + & + \\
\hline O-F test & - & - & + & + & + & + & + & + & + & + & + & + & + & - & + & + & + & + & + & + & - & + & - & + & + & + & + & + & + & + & + & - & - & + & . & + & + & + & + & + & - \\
\hline
\end{tabular}


study revealed that isolates A2 (a, b) could be Klebsiella species because they are catalasepositive, they are non-motile, they are oxidase-negative, they are gram-negative rods, and they are fermenters.

\section{Conclusion}

A total of 61 slopes were inoculated and labeled from soil samples collected at the study site. After pure colonies were obtained by sub-culturing, the study revealed that Streptomycetes species; Escherichia coli; Bacillus species; Enterobacter species; Pseudomonas species, and Klebsiella species were tentatively identified. The suspected Streptomycetes identified were considered as potential organic matter decomposers in arable soil and actively participate in the bioremediation process. Streptomycetes are microbiologically characterized as gram-positive aerobic bacteria that take part in the decomposition of organic compounds. Therefore, they constitute an integral part of the soil microbial population that participates in the bioremediation process.

\section{Acknowledgment}

The authors acknowledge with gratitude contributions of Prof A.N. Okere, Dr. M. Blankson, and Dr. A.A. Amaechi. This study did not receive any grant.

\section{References}

Ahmad, F., Ahmad, I., \& Khan, M. S. (2008). Screening of free-living rhizospheric bacteria for their multiple plant growth-promoting activities. Microbiological Research, 163(2), 173-181.

Alvarez, M. A., Gagne, S. T., \& Antoun, H. (1996). Effect of Compost on Rhizosphere Microflora on the Tomato and on the Incidence of Plant Growth promoting rhizobacteria. Appl Environ Microbiol, 61(1), 194-199.

Anderson, J. M., \& Macfadyen, A. (1975). The role of terrestrial and aquatic organisms in decomposition processes. In The 17th Symposium of the British Ecological Society, 15-18 April 1975.

Das, M., \& Adholeya, A. (2012). Role of Microorganisms in Remediation of Contaminated Soil. In T. Satyanarayana, B. N. Johri, \& A. Prakash (Eds.), Microorganisms in environmental management: microbes and environment. Springer Science \& Business Media.

Egwuatu, T. F., \& Appeh, O. G. (2018). Isolation and Isolation and characterization of filter paper degrading bacteria from the guts of Coptotermes formosanus. Suan Sunandha Science and Technology Journal, $5(2), 20-26$.

Ekenwosu, J. U. (2019). Post Bioremediation Effect on Soil Fertility using Substrate from Livestock Byproduct. International Annals of Science, 7(1), 33-37.

Huddleston, A. S., Cresswell, N., \& Beringer, J. E. (1997). Molecular Detection of Streptomycin-Producing Streptomycetes in Brazilian Soils. Applied and Environmental Microbiology, 63(4), 1288-1297.

Ite, A. E., \& Ibok, U. J. (2019). Role of Plants and Microbes in Bioremediation of Petroleum Hydrocarbons Contaminated Soils. International Journal of Environmental Bioremediation \& Biodegradation, 7(1), 1-19.

Kumar, M., Prasad, R., Goyal, P., Teotia, P., Tuteja, N., Varma, A., \& Kumar, V. (2017). Environmental biodegradation of xenobiotics: role of potential microflora. In Xenobiotics in the Soil Environment (pp. 
319-334).

Osama, N. M., Ibrahim, M. Z., Ghazi, S. ., Shedeed, Z. ., \& Doaa, M. N. (2018). Remediation of Cadmium Toxicity on Alfalfa (Medicago sativa L.) Using Biochar as a Bioadsorbent, Rhizobium meliloti and Arbuscular Mycorrhizal Fungi as Biofertilizers. Journal of Bioremediation \& Biodegradation, 9, 429.

Srivastava, J., Naraian, R., Kalra, S. J. S., \& Chandra, H. (2014). Advances in microbial bioremediation and the factors influencing the process. International Journal of Environmental Science and technology2, 11(6), 1787-1800.

Venosa, A. D., Suidan, M. T., Wrenn, B. A., Strohmeier, K. L., Haines, J. R., Eberhart, B. L., ... Holder, E. (1996). Bioremediation of an experimental oil spill on the shoreline of Delaware Bay. Environmental science \& technology. Environ. Sci. Technol., 30(5), 1764-1775. 